\title{
Up-Regulation of circRNA hsa_circ_0003074 Expression is a Reliable Diagnostic and Prognostic Biomarker in Patients with Osteosarcoma
}

This article was published in the following Dove Press journal: Cancer Management and Research

\section{Saiyun Lei ${ }^{1}$ \\ Lei Xiang ${ }^{2}$}

'Department of Joint Surgical, The Second Affiliated Hospital of University of South China, Hengyang 42100I, People's Republic of China; ${ }^{2}$ Department of Traumatic Orthopedics, Affiliated Nanhua Hospital, University of South China, Hengyang 421002, People's Republic of China
Correspondence: Lei Xiang

Department of Traumatic Orthopedics, Affiliated Nanhua Hospital, University of South China, Hengyang 421002, People's Republic of China

Email xianglei860305@I63.com
Objective: The purpose of this study was to detect the expression level of circRNA hsa_circ_0003074 in the tissues and peripheral blood of patients with osteosarcoma, and to explore its value in diagnosis and predicting prognosis of patients with osteosarcoma.

Materials and Methods: Tissue samples of osteosarcoma patients for chip sequencing were collected and circRNA hsa_circ_0003074, which was associated with the most obvious differential expression, was selected for qRT-PCR analysis. At the same time, the expression levels of hsa_circ_0003074 in different osteosarcoma cell lines, and in the peripheral blood of osteosarcoma patients before and after chemotherapy or surgery, as well as those in healthy volunteers matched by age and sex were also tested. This study aimed to explore the relationship between hsa_circ_0003074 and clinical characteristics of patients with osteosarcoma and analyzed its impact on patient survival.

Results: High expression of hsa_circ_0003074 was detected in osteosarcoma tissues, peripheral blood and osteosarcoma cell lines, and the expression level of hsa_circ_0003074 in the peripheral blood of patients with osteosarcoma decreased significantly after chemotherapy or surgery. In addition, hsa_circ_0003074 was closely related to clinical characteristics, such as tumor size, lung metastasis, enneking stage, and chemotherapy resistance. The ROC curve showed that hsa_circ_0003074 was a good diagnostic indicator for osteosarcoma, and survival analysis results revealed that high expression of hsa_circ_0003074 affected the survival of patients.

Conclusion: hsa_circ_0003074 is not only highly expressed in the tissues and peripheral blood of osteosarcoma patients but also a reliable diagnostic and prognostic biomarker for osteosarcoma patients.

Keywords: osteosarcoma, circRNA, diagnosis, prognosis, biomarkers

\section{Background}

Osteosarcoma is a type of primary malignant bone tumor that tends to occur in children and adolescents. About $80 \%$ of patients with osteosarcoma are 10-25 years old. ${ }^{1}$ These patients have no typical clinical symptoms at onset and the degree of malignancy is high. In the early stage of disease diagnosis, $20 \%$ of patients may already have distant metastases, ${ }^{2}$ including brain and lung metastases, ${ }^{3}$ which is pernicious and associated with a high mortality rate. With the combined use of chemotherapy and radical resection, the survival rate of osteosarcoma patients has been effectively improved, but the 5-year survival rate is still only $60-70 \%{ }^{2}$ Particularly for patients with local recurrence or metastasis, the prognosis is even 
worse, and the 5-year survival rate is only $30 \%{ }^{4}$ Alkaline phosphatase (ALP) and lactate dehydrogenase (LDH) have been the most common serum markers for the diagnosis of osteosarcoma in the past, ${ }^{5,6}$ but the sensitivity and specificity of these markers are unsatisfactory. Therefore, it is urgent to find specific diagnostic and prognostic indicators for early diagnosis and prognosis prediction of osteosarcoma patients, so that these patients can benefit from a more proactive treatment plan as early as possible to improve their prognosis.

CircRNA is a new type of special non-coding covalently closed circular RNA molecule, with a level of stability that is higher than corresponding linear RNA. Since it can be stably expressed in various tissues and cells, ${ }^{7}$ more and more CircRNA molecules have been identified and used as a clinical diagnostic marker. Studies have shown that the abnormal expression of CircRNAs has an important relationship with the occurrence and development of various tumors, such as cholangiocarcinoma, bladder cancer, breast cancer, and non-small cell lung cancer. ${ }^{8-11}$ In addition, the expression of CircRNAs may also be closely related to the prognosis of cancer patients. Studies have found potential CircRNA biomarkers for hepatocellular carcinoma, ${ }^{12}$ gastric cancer, ${ }^{13}$ and lung adenocarcinoma. ${ }^{14}$ These findings indicate that circRNAs are expected to become new diagnostic and prognostic biomarkers for malignant tumors. However, research on CircRNAs as biomarkers for osteosarcoma is still relatively rare. The purpose of this study was to detect the expression of hsa_circ_0003074 in the tumor tissue and serum of osteosarcoma patients as well as a control group, to evaluate its impact on disease-free survival (PFS) and overall survival (OS), and to explore its use as a marker for early diagnosis and prognosis prediction.

\section{Materials and Methods}

\section{Patient and Clinical Data}

A total of 60 patients with primary osteosarcoma who were treated with the same chemotherapy regimen before surgery and underwent complete resection in our hospital from 2017 to 2019 were included. The chemotherapy regimen for patients with osteosarcoma is as follows: After the diagnosis is confirmed by the pathological examination of the tumor site puncture, the patient undergoes four drugs combined sequential neoadjuvant chemotherapy: methotrexate $8-12 \mathrm{~g} / \mathrm{m}^{2}$, ifosfamide $2-3 \mathrm{~g} /$ $\mathrm{m}^{2} \times 3-5$ days, cisplatin $100 \mathrm{mg} / \mathrm{m}^{2}$, epirubicin $60 \mathrm{mg} / \mathrm{m}^{2}$, every 4 single drugs are sequentially a cycle, and the operation is performed after 1 to 2 cycles. A total of 60 pairs of osteosarcoma tissues and their adjacent tissues were obtained. In addition, we also collected peripheral

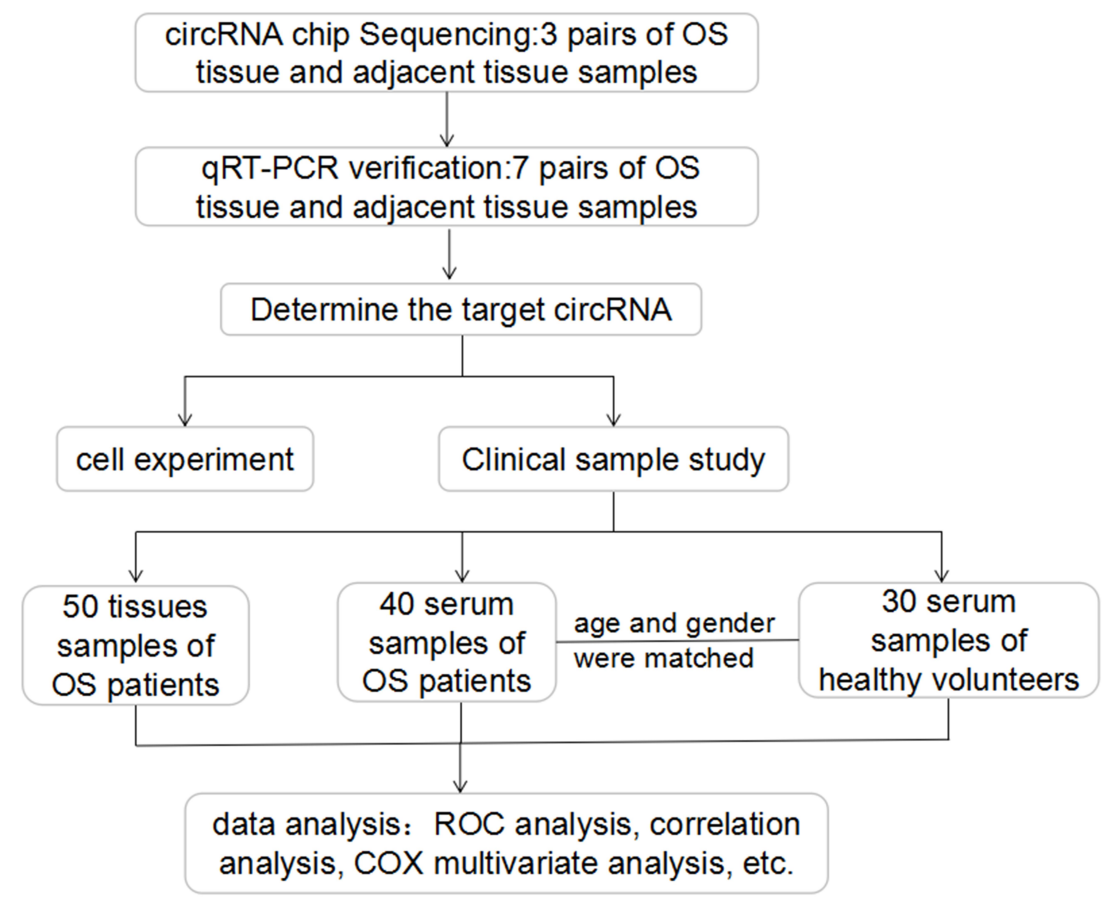

Figure I Flow chart of patient selection in the study and main study procedures. 
Table I Twenty up-regulated and 20 down-regulated circRNA in patients with osteosarcoma

\begin{tabular}{|c|c|c|c|c|c|}
\hline circRNA & Regulation & Fold Change & Chrom & circRNA_Type & P-value \\
\hline hsa_circ_00074 I8 & up & 1.02 & chrl & exonic & 0.01 \\
\hline hsa_circ_0000654 & up & 1.63 & $\mathrm{chr} 15$ & exonic & 0.02 \\
\hline hsa_circ_0007390 & up & 1.05 & $\operatorname{chrl5}$ & exonic & 0.02 \\
\hline hsa_circ_003|460 & up & I.II & $\operatorname{chrl} 4$ & exonic & 0.01 \\
\hline hsa_circ_005545I & up & 1.53 & chr2 & exonic & 0.01 \\
\hline hsa_circ_0007822 & up & 1.29 & chrl & exonic & 0.02 \\
\hline hsa_circ_0005753 & up & 2.71 & chr6 & exonic & 0.03 \\
\hline hsa_circ_000I445 & up & 1.23 & $\operatorname{chr} 4$ & exonic & 0.04 \\
\hline hsa_circ_0002076 & up & 1.54 & chr2 & exonic & 0.02 \\
\hline hsa_circ_0004I03 & up & 2.33 & chrll & exonic & 0.01 \\
\hline hsa_circ_0000566 & up & 1.25 & $\operatorname{chrl} 4$ & exonic & 0.03 \\
\hline hsa_circ_0003074 & up & 3.83 & chrl & exonic & 0.01 \\
\hline hsa_circ_0090868 & up & 2.64 & chrl6 & exonic & 0.03 \\
\hline hsa_circ_0001616 & up & 1.66 & chr6 & exonic & 0.04 \\
\hline hsa_circ_0002863 & up & 1.75 & chr2 & exonic & 0.03 \\
\hline hsa_circ_0007458 & up & 2.55 & chrll & exonic & 0.02 \\
\hline hsa_circ_0035226 & up & 2.35 & chrlo & exonic & 0.04 \\
\hline hsa_circ_000I55I & up & 2.77 & $\operatorname{chr} 5$ & exonic & 0.01 \\
\hline hsa_circ_0004976 & up & 2.22 & chr2 & exonic & 0.03 \\
\hline hsa_circ_0044949 & up & 2.03 & chrl7 & exonic & 0.02 \\
\hline hsa_circ_0047552 & down & 1.24 & chr8 & exonic & 0.03 \\
\hline hsa_circ_00II 933 & down & 1.10 & chrl & exonic & 0.04 \\
\hline hsa_circ_0000546 & down & 1.28 & $\mathrm{chrl} 4$ & exonic & 0.02 \\
\hline hsa_circ_0047/ 35 & down & 1.01 & $\operatorname{chr} 18$ & exonic & 0.01 \\
\hline hsa_circ_0006063 & down & 1.30 & chrlo & exonic & 0.03 \\
\hline hsa_circ_0026134 & down & 1.53 & $\mathrm{chr} / 2$ & exonic & 0.04 \\
\hline hsa_circ_0055377 & down & 1.67 & chr2 & exonic & 0.02 \\
\hline hsa_circ_0075826 & down & 2.71 & chr6 & exonic & 0.01 \\
\hline hsa_circ_0047I5I & down & 1.56 & $\operatorname{chr} 18$ & exonic & 0.03 \\
\hline hsa_circ_0067524 & down & $\mathrm{I} .44$ & chr3 & exonic & 0.04 \\
\hline hsa_circ_0000246 & down & 2.19 & chrlo & exonic & 0.02 \\
\hline hsa_circ_0046753 & down & 1.35 & $\operatorname{chr} 18$ & exonic & 0.04 \\
\hline hsa_circ_004580I & down & 1.61 & chrl7 & exonic & 0.03 \\
\hline hsa_circ_0002554 & down & 1.31 & chr3 & exonic & 0.01 \\
\hline hsa_circ_0019799 & down & 1.68 & chrlo & exonic & 0.0 \\
\hline hsa_circ_0072797 & down & 2.14 & $\operatorname{chr} 5$ & exonic & 0.05 \\
\hline hsa_circ_0049637 & down & 2.66 & chrl9 & exonic & 0.04 \\
\hline hsa_circ_000297I & down & 1.30 & chr6 & exonic & 0.03 \\
\hline hsa_circ_00 I 8758 & down & 2.25 & chrlo & exonic & 0.04 \\
\hline hsa_circ_0075829 & down & 1.45 & chr6 & exonic & 0.01 \\
\hline
\end{tabular}

blood samples from 40 patients with osteosarcoma before and after chemotherapy and surgery as well as that of 30 healthy volunteers matched for age and sex. Once obtained, all tissue samples were stored at $-80{ }^{\circ} \mathrm{C}$ until they were used. Basic information of patients with osteosarcoma (including age, gender, tumor location, Enneking stage, tumor size, lung metastasis, tumor differentiation, chemotherapy resistance, ALP and LDH, etc.) was collected via an electronic medical record system. All patients were followed up. All patients and healthy volunteers provided written informed consent. The study was approved by the ethics committee of our hospital (approve number:2017-S057 and 2017-N039) and was conducted according to the Declaration of Helsinki. Flow chart of patient selection in the study and main study procedures as shown in Figure 1. 
A

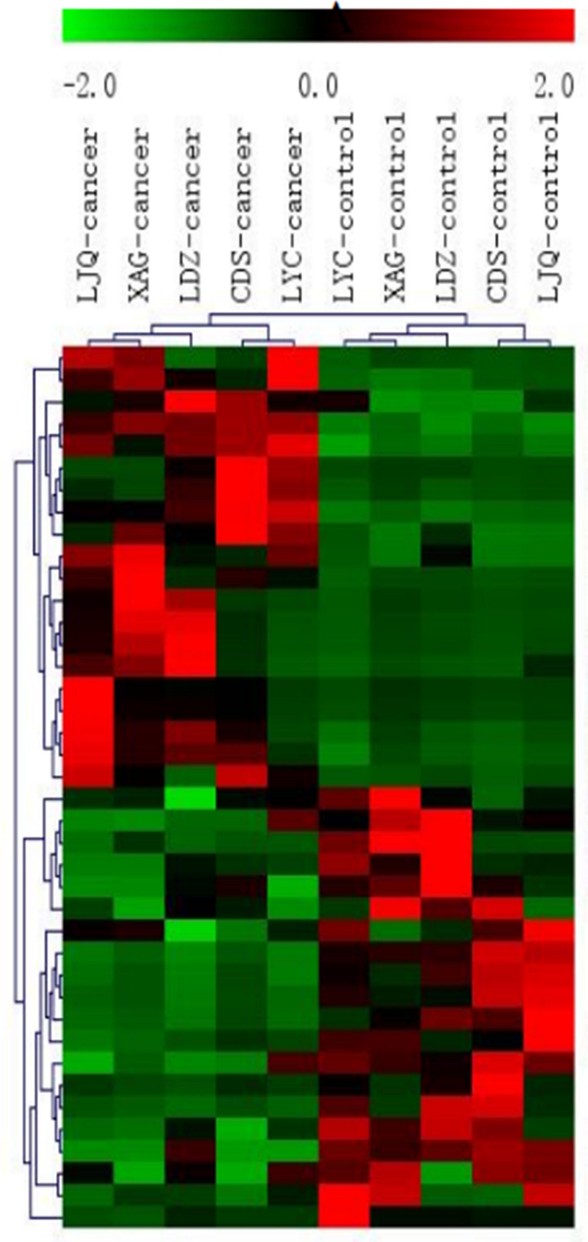

B

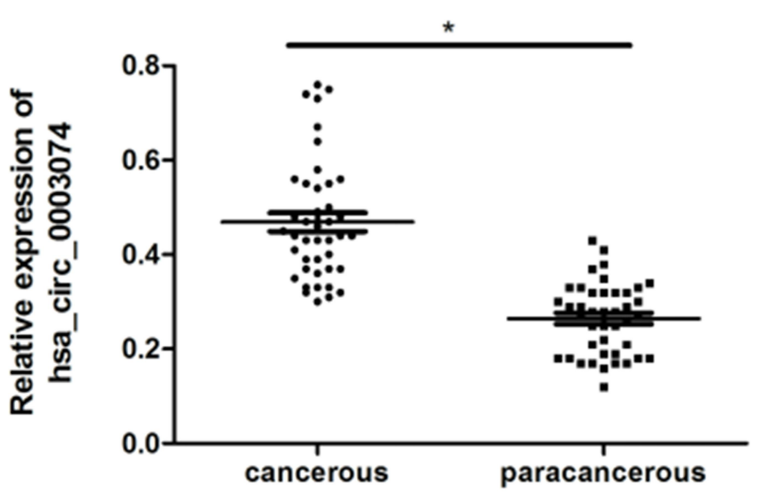

C

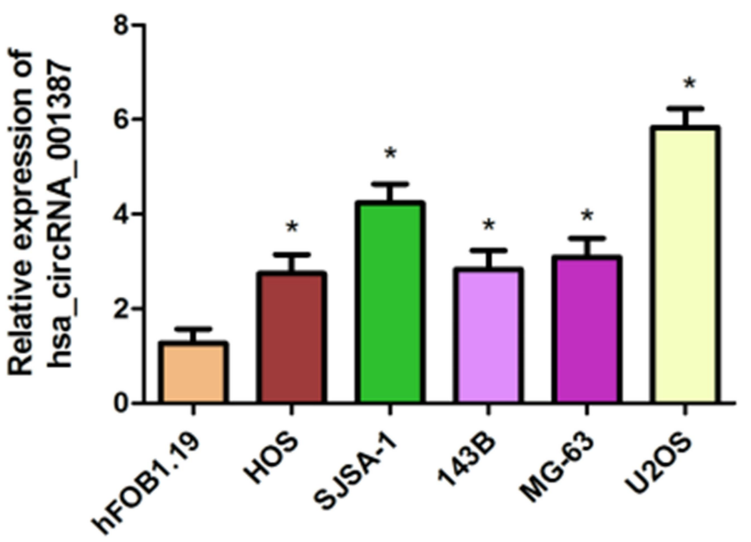

Figure 2 hsa_circ_0003074 expression in osteosarcoma cancer tissues and osteosarcoma cell lines. (A) is a heat map of circRNA chip detection in osteosarcoma cancer tissues and adjacent tissues. (B) shows the results of qRT-PCR detection of hsa circ 0003074 in 60 pairs of osteosarcoma cancer tissues and adjacent tissues. (C) shows the results of qRT-PCR detection of hsa_circ_0003074 in different osteosarcoma cell lines. *The difference is statistically significant compared with adjacent tissues and the hFOBI.19 cell line $(\mathrm{p}<0.05)$, respectively. All experiments are repeated three times.

\section{RNA Extraction and Chip Sequencing}

TRIzol kit (Invitrogen, USA) was used to extract total RNA from osteosarcoma cancer tissues, adjacent tissues and peripheral blood samples. After removing ribosomes and linear RNA, circRNAs were amplified and transcribed into fluorescent cRNA. Arraystar Human circRNA Array V2 (8x15K, Arraystar) was used for sequencing analysis, and GeneSPring 13.0 (Agilent) software was used to analyze circRNA data. Differentially expressed circRNA was defined as a fold change of circRNA $>2.0$ and $P$ value $<0.05$.

\section{Quantitative RT-PCR Verification}

Total RNA was extracted using the TRIzol kit (Invitrogen, USA). After removing the linear RNA from the total RNA, the remaining circRNAs were transcribed into cDNA, and real-time qRT-PCR (Arraystar) was performed using the ViiA 7 Real-time PCR System (APPlied Biosystems). Using GAPDH as a qPCR control, the $\Delta \mathrm{Ct}$ value reflected the circRNA expression level. The gene primers were as follows: hsa_circ_0003074, Forward: 5'-TCCTCTTGTAC GCCCCA-3'; hsa_circ_0003074, Reverse: 5'-GCGGGCAG TCCTCGAGT-3'; GAPDH, Forward: 5'-AATGGGCA GC CGTTAGGAAA-3'; GAPDH, Reverse: 5'-TGAAGGGGT CATTGATG GCA-3'.

\section{Cell Culture}

Normal adult bone cell lines (hFOB1.19) and 5 osteosarcoma cell lines (HOS, SJSA-1, 143B, MG-63 and U2OS) purchased from the Chinese Academy of Sciences (Shanghai) cell bank were cultured in $10 \%$ fetal bovine serum 1640 medium (HyClone, Logan, UT, USA). The 
A

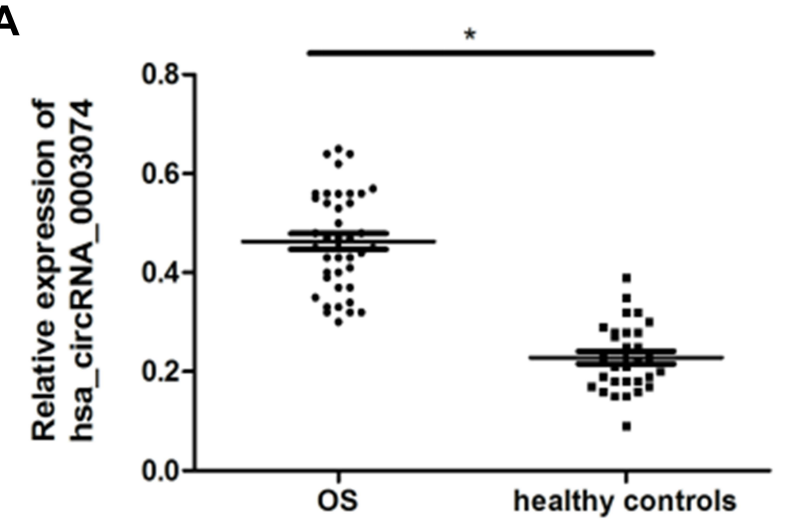

C

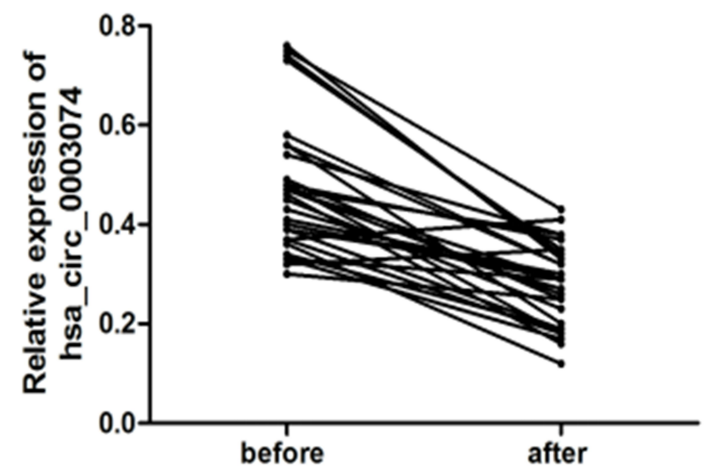

B

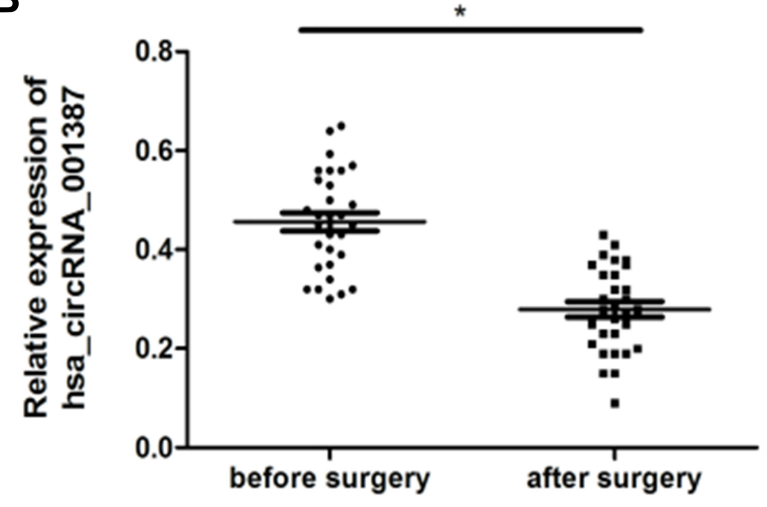

D

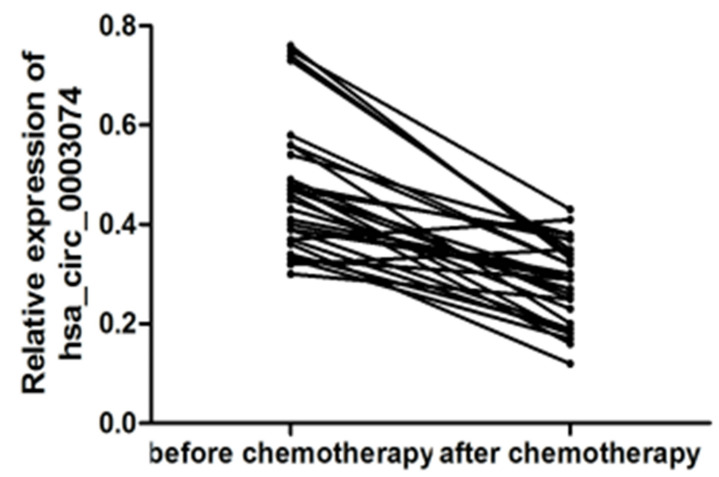

Figure 3 hsa_circ_0003074 expression in the serum of osteosarcoma patients. (A) is the expression of hsa_circ_0003074 in the serum of 40 patients with osteosarcoma and 30 healthy volunteers matched for age and sex. (B) is the expression of hsa_circ_0003074 in serum of 40 patients with osteosarcoma before and after surgery. (C) is the expression of hsa_circ_0003074 in serum of 40 patients with osteosarcoma before and after chemotherapy. (D) is the expression of hsa_circ_0003074 in the serum of osteosarcoma patients who are chemo-sensitive $(n=25)$ and chemo-resistant $(n=15)$. *Indicates that the difference is statistically significant $(p<0.05)$.

medium was placed in a $37^{\circ} \mathrm{C}, 5 \%$ carbon dioxide incubator. Cells at logarithmic growth phase were used for the experiment.

\section{Statistical Analysis}

The data were statistically analyzed using SPSS 20.0 software, and the data were expressed as mean \pm standard deviation. $t$-test or chi-squared test was used to compare whether the differences were statistically significant. A receiver operating curve (ROC) was established, and used to analyze the diagnostic value of hsa_circ_0003074 to osteosarcoma patients. A KaplanMeier survival curve was established, and the Log rank test was used to analyze whether there was a difference in survival rates between high and low circRNA groups. $\mathrm{P}<0.05$ was considered statistically significant.

\section{Results}

High Expression of hsa_circ_0003074 in Osteosarcoma Tissues and Cells

Through chip sequencing, it was found that there were 20 upregulated and 20 down-regulated differentially expressed circRNAs (fold change $>2.0$ and $P$ value $<0.05$ ) in osteosarcoma cancer tissues and adjacent tissues respectively (Table 1), of which hsa_circ_0003074 was most up-regulated (Figure 2A). Subsequently, qRT-PCR was performed on 60 pairs of osteosarcoma cancer tissues and adjacent tissues. The results showed that the expression of hsa_circ_0003074 in cancer tissues increased significantly (Figure 2B), which supported the results of chip sequencing. In addition, the expression of hsa_circ_0003074 was further detected in normal adult bone cell lines (hFOB1.19) and osteosarcoma cell lines (HOS, SJSA-1, 143B, MG-63, and U2OS), and the results showed that HOS, SJSA-1, 143B, MG-63 and U2OS cell lines had 
A

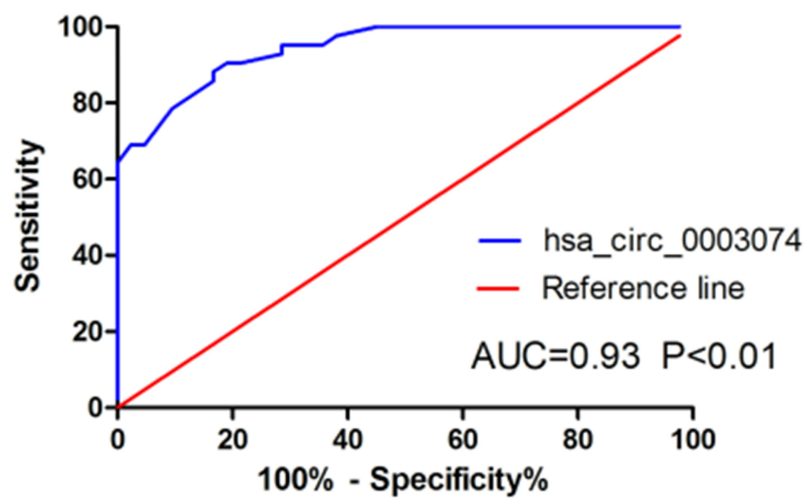

C

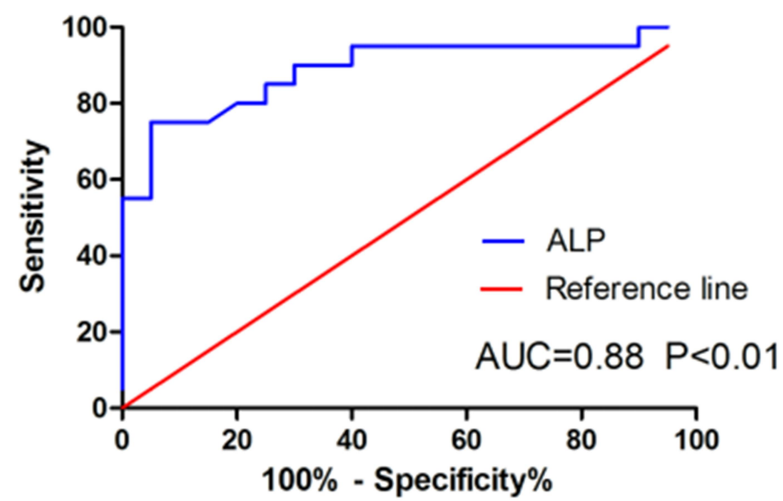

B

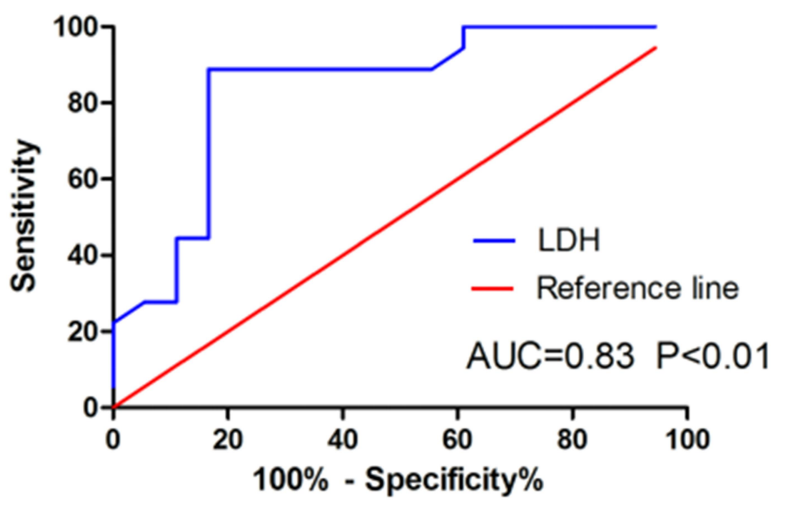

Figure 4 Analysis of hsa_circ_0003074 in the diagnosis of osteosarcoma patients. (A) is a ROC analysis of hsa_circ_0003074 in osteosarcoma patients ( $\mathrm{n}=40$ ) and healthy volunteers $(n=30)$. (B) is a ROC analysis of LDH in osteosarcoma patients $(n=40)$ and healthy volunteers $(n=30)$. (C) is a ROC analysis of ALP in osteosarcoma patients $(n=40)$ and healthy volunteers $(n=30)$.

significantly higher expression of hsa_circ_0003074 than the hFOB1.19 cell line (Figure 2C).

\section{High Expression of hsa_circ_0003074 in the Plasma of Osteosarcoma Patients}

Using qRT-PCR to analyze the expression of hsa_circ_0003074 in the blood samples of 40 patients with osteosarcoma and 30 healthy volunteers matched for age and sex, it was found that compared with healthy volunteers, the expression of hsa_circ_0003074 in the serum of patients with osteosarcoma was substantially higher (Figure 3A). In addition, we also analyzed the expression of hsa_circ_0003074 in the serum of patients with osteosarcoma before and after chemotherapy or surgery. The results showed that the expression of hsa_circ_0003074 in the plasma of patients with osteosarcoma after chemotherapy or after surgery was considerably lower (Figure 3B and C). Also, according to further analysis using the chemotherapy results of patients, the expression of hsa_circ_0003074 in the plasma of chemo-resistant patients was significantly higher (Figure 3D).

\section{hsa_circ_0003074 is Closely Related to the Diagnosis and Prognosis of Osteosarcoma Patients}

A ROC curve was established to analyze the diagnostic value of hsa_circ_0003074 in osteosarcoma, and it was found that hsa_circ_0003074 can clearly distinguish patients with osteosarcoma from healthy volunteers (Figure 4A), with an area under the curve (AUC) of $0.93, \mathrm{P}<0.0001$. In addition, through comparison with the ROC curves of LDH and ALP (Figure 4B and C), it was found that the diagnostic effect of hsa_circ_0003074 in osteosarcoma was better than that of LDH and ALP. The above results indicated that hsa circ_0003074 can be used as an effective diagnostic 
A

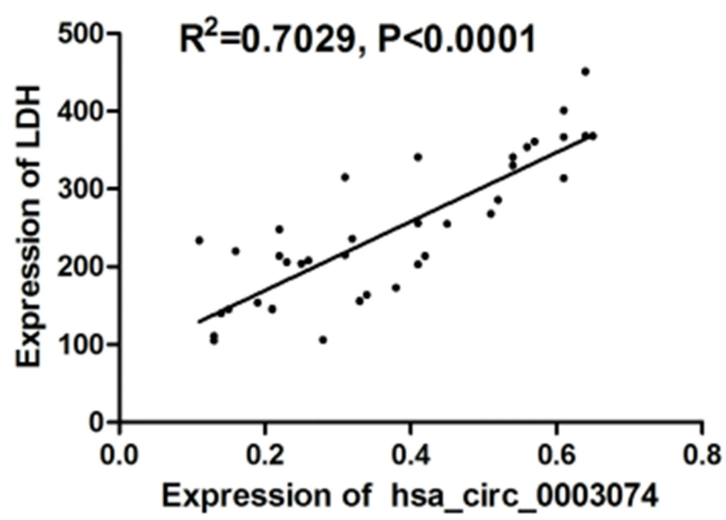

C

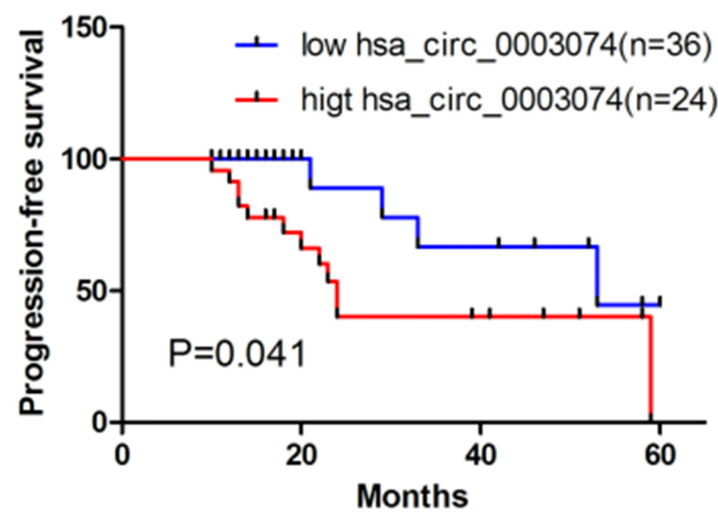

B

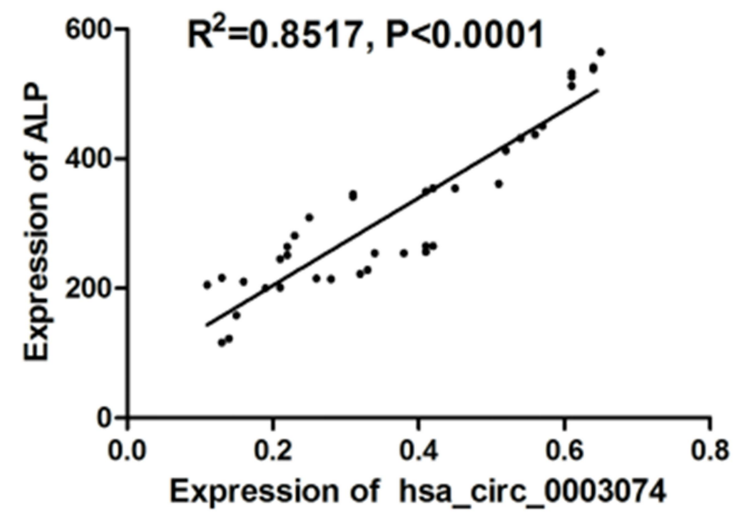

D

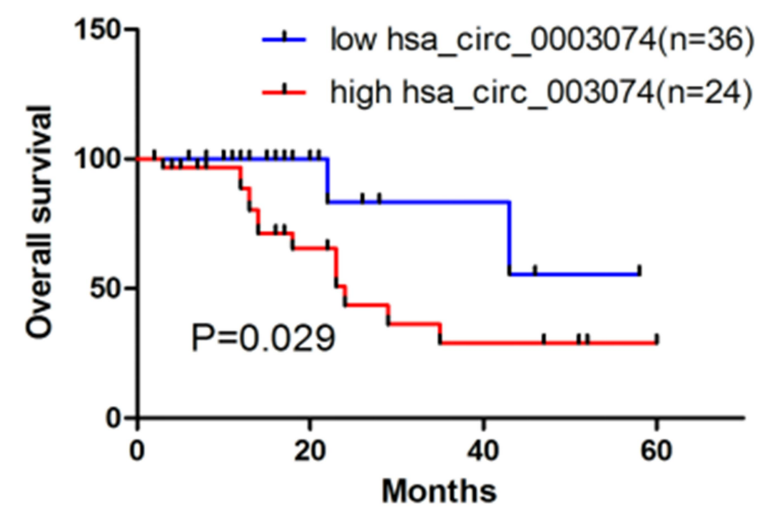

Figure 5 Analysis of hsa_circ_0003074 in the prognosis of osteosarcoma patients. (A and B) show linear regression analysis of hsa_circ_0003074 with LDH and ALP. (C and D) show effects of hsa_circ_0003074 expression levels on the overall survival and progression-free survival of osteosarcoma patients ( $\mathrm{n}=60$ ), respectively.

biomarker in osteosarcoma. In order to analyze the value of hsa_circ_0003074 as a prognostic marker, we first analyzed its correlation with the common clinical prognostic markers ALP and LDH. The results showed that the expression of hsa_circ_0003074 had a strong correlation with ALP and LDH (Figure 5A and B). Then, according to the expression of hsa_circ_0003074 in 60 patients, we divided them into hsa_circ_0003074 high/low expression groups, and established Kaplan-Meier survival curves according to their expression levels. The results showed that OS and PFS of osteosarcoma patients with high expression of hsa_circ_0003074 were significantly lower (Figure 5C and D).

\section{hsa_circ_0003074 is Closely Related to Clinical Symptoms and Severity in Osteosarcoma Patients}

Based on the median (0.465), the level of expression of hsa_circ_0003074 was divided into two groups of high/low expression, and then the relationship between clinicopathological characteristics and hsa_circ_0003074 expression was analyzed. The results showed that the expression level of hsa_circ_0003074 was related to tumor size, lung metastasis, Enneking Stage, resistance to chemotherapy, ALP and LDH, but it was not related to age, gender, tumor differentiation, and surgical site (Table 2). Subsequent further analysis revealed that patients with tumors $\geq 8 \mathrm{~cm}$, lung metastases, and stage IIB-III disease had significantly increased hsa_circ_0003074 expression levels (Figure $6 \mathrm{~A}-\mathrm{C})$. Finally, based on the follow-up results, the relationship between hsa_circ_0003074 and disease recurrence was analyzed. The results showed that the expression level of hsa_circ_0003074 in osteosarcoma patients following relapse was significantly higher than that in patients without relapse (Figure 6D). 
Table 2 Correlation between clinicopathological characteristics of patients with osteosarcoma and expression of hsa_circ_0003074 in cancer tissue

\begin{tabular}{|c|c|c|c|}
\hline & $\begin{array}{l}\text { Low Expression of } \\
\text { hsa_circ_0003074 }\end{array}$ & $\begin{array}{l}\text { High Expression of } \\
\text { hsa_circ_0003074 }\end{array}$ & $P$ value \\
\hline $\begin{array}{l}\text { Gender } \\
\text { male } \\
\text { female }\end{array}$ & $\begin{array}{l}17 \\
11\end{array}$ & $\begin{array}{l}18 \\
14\end{array}$ & 0.293 \\
\hline $\begin{array}{l}\text { Age (years) } \\
\quad<25 \\
\geq 25\end{array}$ & $\begin{array}{l}15 \\
16\end{array}$ & $\begin{array}{l}18 \\
11\end{array}$ & 0.314 \\
\hline $\begin{array}{l}\text { Enneking stage } \\
\text { stage I-IIA } \\
\text { stage IIB-III }\end{array}$ & $\begin{array}{l}18 \\
10\end{array}$ & $\begin{array}{l}7 \\
25\end{array}$ & $<0.01$ \\
\hline $\begin{array}{l}\text { Tumor size }(\mathrm{cm}) \\
\quad<8 \\
\geq 8\end{array}$ & $\begin{array}{l}17 \\
11\end{array}$ & $\begin{array}{l}5 \\
27\end{array}$ & $<0.01$ \\
\hline $\begin{array}{l}\text { Surgical site } \\
\text { Femur } \\
\text { Tibia } \\
\text { other }\end{array}$ & $\begin{array}{l}11 \\
14 \\
5\end{array}$ & $\begin{array}{l}15 \\
12 \\
3\end{array}$ & 0.308 \\
\hline $\begin{array}{l}\text { Differentiation } \\
\text { High } \\
\text { Moderate } \\
\text { Low }\end{array}$ & $\begin{array}{l}5 \\
10 \\
14\end{array}$ & $\begin{array}{l}8 \\
13 \\
10\end{array}$ & 0.073 \\
\hline $\begin{array}{l}\text { Lung metastasis } \\
\text { Yes } \\
\text { No }\end{array}$ & $\begin{array}{l}8 \\
18\end{array}$ & $\begin{array}{l}25 \\
9\end{array}$ & $<0.01$ \\
\hline $\begin{array}{l}\text { Chemoresistant } \\
\text { Yes } \\
\text { No }\end{array}$ & $\begin{array}{l}9 \\
19\end{array}$ & $\begin{array}{l}22 \\
10\end{array}$ & $<0.01$ \\
\hline $\begin{array}{l}\text { LDH } \\
\qquad \text { Normal } \\
\text { Abnormal }\end{array}$ & $\begin{array}{l}19 \\
11\end{array}$ & $\begin{array}{l}12 \\
18\end{array}$ & 0.043 \\
\hline $\begin{array}{l}\text { ALP } \\
\qquad \text { Normal } \\
\text { Abnormal }\end{array}$ & 18 & $\begin{array}{l}9 \\
20\end{array}$ & 0.041 \\
\hline
\end{tabular}

Abbreviations: LDH, lactate dehydrogenase; ALP, alkaline phosphatase.

\section{hsa_circ_0003074 is an Independent Factor that Predicts Prognosis of Osteosarcoma Patients}

The factors that can predict prognosis in patients with osteosarcoma were studied using univariate and multivariate analysis. Univariate analysis showed that hsa_circ_0003074 expression levels, Enneking Stage, tumor size, lung metastasis, chemotherapy resistance, ALP and LDH were significantly correlated with the prognosis of osteosarcoma patients
(Table 3). The above factors were further included in multivariate analysis. The results showed that hsa_circ_0003074 expression levels, Enneking Stage, tumor size, lung metastasis, and chemotherapy resistance were independent factors that were predictive of prognosis of osteosarcoma patients (Table 3).

\section{Discussion}

Osteosarcoma is a common bone tumor in China, associated with a high level of malignancy, and the incidence is about 3/1 million/year. ${ }^{15}$ Osteosarcoma has no obvious precancerous lesions. At the point of diagnosis, the disease is usually advanced and metastatic, with a poor prognosis. $^{16,17}$ In addition, chemotherapy resistance and lung metastasis have always been important factors affecting the prognosis of osteosarcoma patients. ${ }^{18}$ Therefore, identifying markers to facilitate early diagnosis and prognosis prediction in osteosarcoma patients is important for improving prognosis of osteosarcoma patients and improving their quality of life. As a focal point of research in recent years, circRNAs have been shown to have an increased level of expression in many diseases and, as such, can be used as markers for early diagnosis, especially in tumors. ${ }^{19-21}$ In this study, it was found for the first time that the expression of circRNA hsa_circ_0003074 was increased in osteosarcoma patients, and that it could be used as a reliable biomarker for early diagnosis and prognosis in osteosarcoma patients.

CircRNAs are conservative and stable circular molecules that can be stably expressed in many diseases (especially tumors), including osteosarcoma. Zhu Kun-Peng et $\mathrm{al}^{22}$ found that hsa_circ_0081001 was highly expressed in osteosarcoma tissues, Zhu Kai et $\mathrm{al}^{23}$ showed that hsa_circ_0000885 was highly expressed in the serum of osteosarcoma patients, and Qi and his colleagues ${ }^{24}$ found that circ_0000502 was significantly up-regulated in osteosarcoma and could predict poor prognosis of osteosarcoma patients. In this study, it was found that hsa_circ_0003074 was highly expressed in the cancer tissues and serum of osteosarcoma patients and in osteosarcoma cell lines. In addition, it was also found that hsa_circ_0003074 in the serum of patients with osteosarcoma following chemotherapy and surgery was significantly decreased. This result is consistent with the results of Zhu Kai et al, suggesting treatment methods such as chemotherapy and surgery may affect circRNA expression. Subsequently, further analysis of the expression of hsa_circ_0003074 and the clinicopathological characteristics of patients 

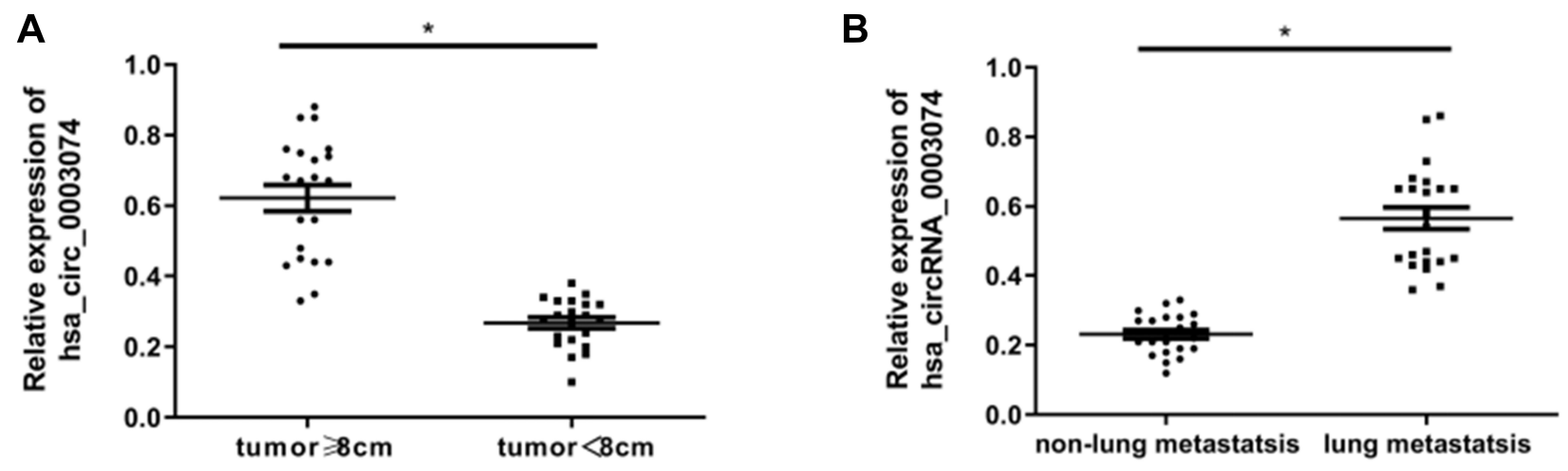

C
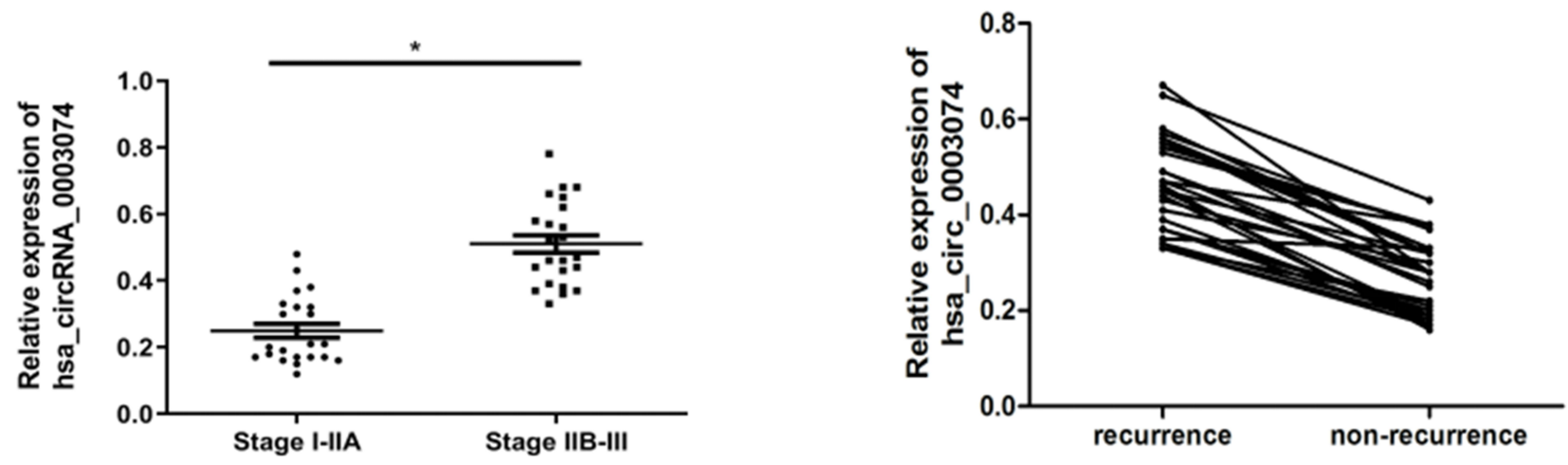

Figure 6 Correlation analysis of hsa_circ_0003074 and clinicopathological characteristics in patients with osteosarcoma. (A) is the expression of hsa_circ_0003074 in the serum of osteosarcoma patients $(n=40)$ with different tumor sizes. $(B)$ is the expression of hsa_circ_0003074 in the serum of osteosarcoma patients $(n=40)$ with lung metastasis and non-pulmonary metastasis. (C) is the expression of hsa_circ_0003074 in the serum of osteosarcoma patients ( $n=40)$ with different Enneking stages. (D) is the expression of hsa_circ_0003074 in the serum of osteosarcoma patients $(n=40)$ with and without relapse. *Indicates that the difference is statistically significant $(p<0.05)$.

with osteosarcoma revealed that patients with tumors $\geq 8 \mathrm{~cm}$, lung metastasis, Enneking stage IIB-III, and chemotherapy resistance had high levels of hsa_circ_0003074 expression, and the condition of these patients was usually more severe, with worse prognosis. Therefore, these findings suggest that the expression level of hsa_circ_0003074 can determine the severity of disease in patients with osteosarcoma.

Table 3 Univariate and Multivariate analysis of prognostic factors of patients with osteosarcoma

\begin{tabular}{|c|c|c|c|c|c|c|}
\hline \multirow[t]{2}{*}{ Variables } & \multicolumn{3}{|c|}{ Univariate Analysis } & \multicolumn{3}{|c|}{ Multivariate Analysis } \\
\hline & Hazard Ratio & $95 \% \mathrm{Cl}$ & $P$ value & Hazard Ratio & $95 \% \mathrm{Cl}$ & $P$ value \\
\hline Age & 0.943 & $0.345-1.244$ & 0.532 & & & \\
\hline Sex & 0.895 & $0.724-0.938$ & 0.634 & & & \\
\hline Tumor size & 2.483 & $1.114-2.945$ & 0.034 & 3.633 & $2.414-4.386$ & 0.001 \\
\hline Differentiation & 1.473 & $1.023-2.473$ & 0.336 & & & \\
\hline Enneking Stage & 2.394 & $1.734-3.398$ & 0.025 & 3.877 & $1.335-5.394$ & 0.003 \\
\hline Lung metastasis & 3.545 & $2.235-4.325$ & 0.013 & 4.862 & $3.548-5.521$ & 0.003 \\
\hline Surgical site & 1.053 & $1.011-1.565$ & 0.436 & & & \\
\hline Chemoresistant & 2.532 & $1.624-3.334$ & 0.033 & 3.957 & $2.454-4.601$ & 0.003 \\
\hline LDH & 1.837 & I.165-2.657 & 0.043 & & & \\
\hline ALP & 1.953 & $1.212-2.943$ & 0.042 & & & \\
\hline hsa_circ_0003074 & 3.725 & $1.906-5.565$ & 0.011 & 4.927 & $3.954-5.625$ & 0.001 \\
\hline
\end{tabular}

Abbreviations: LDH, lactate dehydrogenase; ALP, alkaline phosphatase. 
ALP and LDH are currently effective blood markers for evaluating the prognosis of osteosarcoma patients. Previous studies have found that patients with metastatic osteosarcoma have significantly increased ALP, and increased ALP can be used as a prognostic indicator of osteosarcoma. ${ }^{25} \mathrm{LDH}$ can reflect tumor load via participation in glycolysis, and it has been shown that the expression level of LDH is closely related to the prognosis of patients with osteosarcoma. ${ }^{26}$ In this study, it was first found by ROC analysis that the diagnostic sensitivity and specificity of hsa_circ_0003074 were higher than those of LDH and ALP. Secondly, through correlation analysis, it was found that the expression of hsa_circ_0003074 had a strong correlation with the expression of ALP and LDH, which indirectly showed that hsa_circ_0003074 may be related to the prognosis of patients with osteosarcoma. Next, we further found through survival analysis that OS and FPS of osteosarcoma patients with high expression of hsa_circ_0003074 were significantly decreased. Finally, through univariate and multivariate analysis, it was shown that hsa_circ_0003074 was an independent prognostic factor of osteosarcoma patients. These results indicated that hsa_circ_0003074 can effectively predict the prognosis of patients with osteosarcoma.

In conclusion, the results of this study indicate that hsa_circ_0003074 is highly expressed in osteosarcoma cancer tissues and serum, and is related to poor prognosis of the patients. hsa_circ_0003074 can be used as an effective diagnostic and prognostic biomarker.

\section{Ethics Approval and Informed Consent}

This study was approved by the Ethics Committee of The second affiliated hospital of University of South China and the First affiliated Nanhua hospital of University of South China. Informed consent was obtained from each subject in accordance with the Declaration of Helsinki.

\section{Author Contributions}

All authors contributed towards data analysis, drafting and critically revising the paper, gave final approval of the version to be published, and agreed to be accountable for all aspects of the work.

\section{Disclosure}

The authors report no conflicts of interest for this work.

\section{References}

1. Bielack SS, Kempf-Bielack B, Delling G, et al. Prognostic factors in high-grade osteosarcoma of the extremities or trunk: an analysis of 1702 patients treated on neoadjuvant cooperative osteosarcoma study group protocols. J Clin Oncol. 2002;20(3):776-790. doi:10.1200/ JCO.2002.20.3.776

2. Bacci G, Bertoni F, Longhi A, et al. Neoadjuvant chemotherapy for high-grade central osteosarcoma of the extremity. Histologic response to preoperative chemotherapy correlates with histologic subtype of the tumor. Cancer. 2003;97(12):3068-3075. doi:10.1002/cncr.11456

3. Shweikeh F, Bukavina L, Saeed K, et al. Brain metastasis in bone and soft tissue cancers: a review of incidence, interventions, and outcomes. Sarcom. 2014;47:51-75.

4. Arndt CA, Rose PS, Folpe AL, et al. Common musculoskeletal tumors of childhood and adolescence. Mayo Clin Proc. 2012;87 (5):475-487. doi:10.1016/j.mayocp.2012.01.015

5. Fu Y, Lan T, Cai H, et al. Meta-analysis of serum lactate dehydrogenase and prognosis for osteosarcoma. Medicine. 2018;97(19): e0741. doi:10.1097/MD.0000000000010741

6. Marais LC, Bertie J, Rodseth R, et al. Pre-treatment serum lactate dehydrogenase and alkaline phosphatase as predictors of metastases in extremity osteosarcoma. $J$ Bone Oncol. 2015;4(3):80-84. doi:10.1016/j.jbo.2015.09.002

7. Meng S, Zhou H, Feng Z, et al. Circ RNA: functions and properties of a novel potential biomarker for cancer. Mol Cancer. 2017;16 (1):94. doi:10.1186/s12943-017-0663-2

8. Xu Y, Yao Y, Zhong X, et al. Downregulated circular RNA hsa_circ_0001649 regulates proliferation, migration and invasion in cholangiocarcinoma cells. Biochem Biophys Res Commun. 2018;496 (2):455-461. doi:10.1016/j.bbrc.2018.01.077

9. Li P, Yang X, Yuan W, et al. CircRNA-Cdrlas exerts anti-oncogenic functions in bladder cancer by sponging microRNA-135a. Cell Physiol Biochem. 2018;46(4):1606-1616. doi:10.1159/000489208

10. Song L, Xiao Y. Downregulation of hsa_circ_0007534 suppresses breast cancer cell proliferation and invasion by targeting miR-593/ MUC19 signal pathway. Biochem Biophys Res Commun. 2018;503 (4):2603-2610. doi:10.1016/j.bbrc.2018.08.007

11. Li Y, Hu J, Li L, et al. Upregulated circular RNA circ_0016760 indicates unfavorable prognosis in NSCLC and promotes cell progression through miR-1287/GAGE1 axis. Biochem Biophys Res Commun. 2018;503(3):2089-2094. doi:10.1016/j.bbrc.2018.07.164

12. Lei K, Bai H, Wei Z, et al. The mechanism and function of circular RNAs in human diseases. Exp Cell Res. 2018;368(2):147-158. doi:10.1016/j.yexcr.2018.05.002

13. Chen S, Zhao Y. Circular RNAs: characteristics, function, and role in human cancer. Histol Histopathol. 2018;33(9):887-893. doi:10.14 670/HH-11-969

14. Zhang M, Xin Y. Circular RNAs: a new frontier for cancer diagnosis and therapy. J Hematol Oncol. 2018;11(1):21. doi:10.1186/s13045018-0569-5

15. Picci P. Osteosarcoma (osteogenic sarcoma). Orphanet J Rare Dis. 2007;2(6):6. doi:10.1186/1750-1172-2-6

16. Kager L, Zoubek A, Pötschger U. Primary metastatic osteosarcoma: presentation and outcome of patients treated on neoadjuvant cooperative osteosarcoma study group protocols. J Clin Oncol. 2003;21 (10):2011-2018. doi:10.1200/JCO.2003.08.132

17. Sampo M, Koivikko M, Taskinen M, et al. Incidence, epidemiology and treatment results of osteosarcoma in Finland-a nationwide population-based study. Acta Oncol. 2011;50(8):1206-1214. doi:10. 3109/0284186X.2011.615339

18. Zhou W, Hao M, Du X, et al. Advances in targeted therapy for osteosarcoma. Discov Med. 2014;17(96):301-307.

19. Qin M, Liu G, Huo X, et al. Hsa_circ_0001649: a circular RNA and potential novel biomarker for hepatocellular carcinoma. Cancer Biomark. 2016;16(1):161-169. doi:10.3233/CBM-150552 
20. Chen S, Li T, Zhao Q, et al. Using circular RNA hsa circ 0000190 as a new biomarker in the diagnosis of gastric cancer. Clin Chim Acta. 2017;466:167-171. doi:10.1016/j.cca.2017.01.025

21. Zhu X, Wang X, Wei S, et al. hsa circ 0013958: a circular RNA and potential novel biomarker for lung adenocarcinoma. FEBS J. 2017;284(14):2170-2182. doi:10.1111/febs.14132

22. Zhu KP, Zhang CL, Hu JP, et al. A novel circulating hsa_circ_0081001 act as a potential biomarker for diagnosis and prognosis of osteosarcoma. Int J Biol Sci. 2018;14(11):1513-1520. doi:10.7150/ijbs. 27523

23. Zhu K, Niu L, Wang J, et al. Circular RNA hsa circ 0000885 levels are increased in tissue and serum samples from patients with osteosarcoma. Med Sci Monit. 2019;25:1499-1505. doi:10.12659/ MSM.914899
24. Qi H, Sun Y, Jiang Y, et al. Upregulation of circular RNA circ_0000502 predicts unfavorable prognosis in osteosarcoma and facilitates cell progression via sponging miR-1238. J Cell Biochem. 2019;120(5):8475-8482. doi:10.1002/jcb.28134

25. Moss DW. Perspectives in alkaline phosphatase research. Clin Chem. 1992;38(12):2486-2492. doi:10.1093/clinchem/38.12.2486

26. Chen J, Sun MX, Hua YQ, et al. Prognostic significance of serum lactate dehydrogenase level in osteosarcoma: a meta-analysis. J Cancer Res Clin. 2014;140(7):1205-1210. doi:10.1007/s00432014-1644-0

\section{Publish your work in this journal}

Cancer Management and Research is an international, peer-reviewed open access journal focusing on cancer research and the optimal use of preventative and integrated treatment interventions to achieve improved outcomes, enhanced survival and quality of life for the cancer patient.
The manuscript management system is completely online and includes a very quick and fair peer-review system, which is all easy to use. Visit http://www.dovepress.com/testimonials.php to read real quotes from published authors. 